\title{
RADON TRANSFORM ON AFFINE BUILDINGS OF RANK THREE
}

\author{
LAURA ATANASI
}

(Received 2 September 1996; revised 5 September 1998)

Communicated by G. Robertson

\begin{abstract}
We define the Radon transform for functions on the set of chambers of affine, locally finite, rank three buildings. We investigate the problem of the inversion of this transform. Explicit inversion formulas are exhibited for functions which fulfill required summability conditions.
\end{abstract}

1991 Mathematics subject classification (Amer. Math. Soc.): primary 51E24, 44A12.

\section{Introduction}

An affine building of rank three is a building $\mathbf{X}$ whose diagram is one of the following:

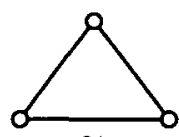

$\tilde{A}_{2}$
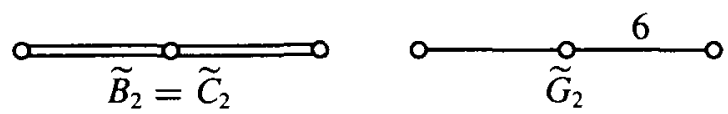

FigurE 1.

We denote by $\mathscr{C}$ the collection of 2-simplices (or chambers) in $\mathbf{X}$ and by $\mathscr{A}$ the collection of apartments in $\mathbf{X}$. Given a function $f$ on $\mathscr{C}$ its Radon transform $R f$ is the function on $\mathscr{A}$ given by

$$
R f(A)=\sum_{c \in A} f(c) \text { for each } A \in \mathscr{A}
$$

(C) 1999 Australian Mathematical Society 0263-6115/99 \$A2.00+0.00 
provided that the series converges absolutely.

In analogy with the setting of $\mathbb{R}^{n}$ or of an affine building of rank two $([1,4])$ we study the problem of recovering $f$ from $R f$ or in other words of inverting $R$. It will be shown that an inversion formula can be obtained by applying to $R$ an integral operator which is obtained by radializing, with respect to a measure $m$, defined on $\mathscr{A}$, a discrete inversion formula obtained case by case with geometric-combinatoric methods. The difficulties are related to the complicated geometry of buildings but two known facts simplify the matter. The first is that each apartment is a retract of the building, hence much work can be done by means of the combinatorics of the affine Coxeter group of the building. The second is related to the local geometry of affine buildings : for each simplex there is a spherical finite building to which we can project the whole affine building.

The author wishes to thank Professors M. Picardello, E. Casadio Tarabusi, and T. Steger for their suggestions during the period she was working at her doctoral thesis at the University of Rome 'La Sapienza'. The author is indebted to T. Steger for his contribution after the referee's report, which improved the previous version of this work.

\section{Preliminaries}

The basic notation is standard and we refer to the books [6,9] and [11] for more details. We shall assume familiarity with elementary facts about Coxeter groups and Coxeter complexes (see also [8] and [7]).

In a rank-three affine building $\mathbf{X}$ a typical apartment arises from a tiling of the Euclidean plane. Each chamber can be identified with a triangle having angles $\pi / k$, $\pi / l, \pi / m$ where $(k, l, m)=(3,3,3),(2,4,4),(2,3,6)$ in the respective cases $\widetilde{A_{2}}$, $\widetilde{B}_{2}, \widetilde{G_{2}}$. (Notice that other authors would call this kind of building 'rank-two'; by rank we mean the number of vertices of any chamber.) Each vertex of $\mathbf{X}$ is labelled with a type which is an integer in $I=\{0,1,2\}$, and each chamber has exactly one vertex of each type. A 1 -simplex (or edge) $\sigma$ has cotype $i$, (that is type $I \backslash\{i\}$ ), if the set of labels associated with the vertices of $\sigma$ is $I \backslash\{i\}$ ). We always suppose $\mathbf{X}$ to be thick (that is each edge is contained in at least three chambers), in which case all edges of a given cotype $i$ are contained in the same number of chambers $q_{i}+1$.

Any two simplices $\sigma, \sigma^{\prime}$ in the building are contained in a common apartment. Furthermore if $A$ and $A^{\prime}$ are apartments with a common chamber then there exists a unique isomorphism between $A$ and $A^{\prime}$ fixing $A \cap A^{\prime}$ pointwise, [6, p. 77]. Given two chambers $c$ and $c^{\prime}$, a gallery of extremes $c$ and $c^{\prime}$ and length $n$ is a finite sequence $\left(c=c_{0}, c_{1}, \ldots, c_{n}=c^{\prime}\right.$ ) where $c_{i}$ and $c_{i+1}$, for $i=0, \ldots, n-1$, are adjacent (that is they have a common edge); if $c_{i} \neq c_{i+1}$ for all $i$, we say that the gallery is simple. 
There always exists a non-unique shortest such gallery, called minimal; the distance between two chambers $c$ and $c^{\prime}$, denoted by $\mathbf{d}\left(c, c^{\prime}\right)$, is the length of a minimal gallery connecting them. More generally the distance between a chamber $c$ and a simplex $\sigma$ in the building is the minimal length of a gallery $\left(c_{0}, c_{1}, \ldots, c_{n}\right)$ with $c_{0}$ containing $\sigma$ and $c_{n}=c$. A gallery which achieves this minimum is called a stretched gallery from $\sigma$ to $c$.

Two chambers $c$ and $c^{\prime}$ which have a common edge of cotype $i, i \in l$, are called $i$-adjacent, and denoted by $c \sim c_{i}^{\prime}$. As two distinct adjacent chambers are $i$-adjacent for a unique $i$, a simple gallery $\left(c_{0}, c_{1}, \ldots, c_{n}\right)$ has a well defined type $\left(i_{0}, \ldots, i_{n-1}\right)$ such that $c_{j} \widetilde{i}_{i_{j}} c_{j+1}$ for $j=0, \ldots, n-1$.

The diagram of the building is the diagram of its (affine) Coxeter group $W$. Fixing an apartment $A$ in the building, and a chamber $c \in A, W$ is generated by the set $S$ of reflections with respect to the edges of $c$ (or simple reflections). The group $W$ acts (on the left) on $A$ by simplicial type-preserving automorphism (with $c$ as a fundamental domain for this action) and its action is simply transitive on the set of chambers (indeed the stabilizer of $c$ is the trivial subgroup). If for each subset $\mathbf{X}^{\prime}$ of $\mathbf{X}$ we denote by $\mathscr{C}\left(\mathbf{X}^{\prime}\right)$ the set of chambers of $\mathbf{X}^{\prime}$, then it follows that the set $\mathscr{C}(A)$ can be identified with $W$, via $w c \rightarrow w$. Moreover, if we label by $s_{i}, i=0,1,2$, the reflection with respect to the edge of $c$ of cotype $i$, two distinct elements $w, w^{\prime} \in W$ correspond to $i$-adjacent chambers if and only if $w^{\prime}=w s_{i}$. Let $l(w)$, or $l_{S}(w)$ if necessary, denote the length of $w$ with respect to $S$. In view of the previous paragraphs, simple galleries starting at $c$ are in 1-1 correspondence with $S$-words. Under this correspondence minimal galleries correspond to reduced words. Then it follows that the metric spaces $\mathscr{C}(A)$ and $W$ are isometric, where the latter is endowed with the word distance.

A reflection $r$ in $W$ is a conjugate of some $s \in S$. The set $M r$ of all simplices in $A$ fixed by $r$ is a subcomplex of dimension one and is called the wall (in $A$ ) determined by $r$. Each wall partitions $\mathscr{C}(A)$ into two parts $\Phi_{r}^{+}$and $\Phi_{r}^{-}$(with $c \in \Phi_{r}^{+}$) interchanged by $r$. These two parts of $\mathscr{C}(A)$ are called half-apartments determined by the wall $M_{r}$. They form complementary subsets of $\mathscr{C}(A)$ and are said to be opposite to each other. As subsets of $W, \Phi_{r}^{+}=\{w \in W: l(r w)>l(w)\}$ and $\Phi_{r}^{-}=W-\Phi_{r}^{+}=\{w \in W: l(r w)<l(w)\}$. The boundary $\partial \Phi_{r}=\Phi_{r}^{+} \cap \Phi_{r}^{-}$of $\Phi_{r}^{+}$ (or of $\Phi_{r}^{-}$) is the wall $M_{r}$. By the interior of a half-apartment we mean its topological interior. There are bijective correspondences between the set of reflections, the set of walls and the set of pairs of opposite half-apartments [9, Proposition 2.6].

There is an obvious action of $W$ on the set of walls of an apartment and then an action of $W$ on the set of half-apartments. For each $w \in W$ the number of positive half-apartments that $w$ maps to negative one is equal to the length of $w$ [8, Proposition 5.6]. By definition two walls are parallel if their distance (that is the distance from any point of one to the nearest point of the other) is bounded. A necessary and sufficient condition for two walls $\partial \Phi_{r}$ and $\partial \Phi_{t}$ to be parallel is that the element $r t$ has infinite 
order [6, p. 142]. As there exists a bijective correspondence between the set $I$ of types of $\mathbf{X}$ and the set $S$ of generators of $W$ (via $i \rightarrow s_{i}$ ) sometimes in the sequel for each reflection $r=s_{i_{1}} s_{i_{2}} \cdots s_{i_{k}}$ we will write $\Phi_{i_{1} i_{2} \cdots i_{k}}^{ \pm}$for $\Phi_{r}^{ \pm}$. A wall or a half-apartment in $\mathbf{X}$ is by definition a wall or a half-apartment in some apartment of $\mathbf{X}$.

Let us call a chamber subcomplex $\mathbf{X}^{\prime}$ of $\mathbf{X}$ convex if any minimal gallery between two chambers of $\mathbf{X}^{\prime}$ lies entirely in $\mathbf{X}^{\prime}$. The convex hull of any subset $\mathbf{X}^{\prime}$, which we denote by conv $\mathbf{X}^{\prime}$, is by definition the intersection of all convex chamber subcomplexes containing $\mathbf{X}^{\prime}$.

Let $\sigma$ be any simplex in $\mathbf{X}$ and set $\mathscr{A}_{\sigma}=\{A \in \mathscr{A}: \sigma \in A\}$. Denote by $l k(\sigma)$ the set of all simplices in $\mathbf{X}$ containing $\sigma$ (with the inclusion order relation induced by that of $\mathbf{X}$ ). It turns out that $l k(\sigma)$ is a spherical (finite) building whose set of apartments is equal to $\left\{A \cap l k(\sigma): A \in \mathscr{A}_{\sigma}\right\}$. If $\sigma$ has type $I \backslash J(J \subseteq I)$ and if $W_{J}$ is the subgroup of $W$ which is generated by $s_{j}, j \in J$ then the finite Coxeter group $W_{\sigma}$ of $l k(\sigma)$ is equal to $W_{J}$. In terms of diagrams this has the following interpretation. Let us recall that the diagram $M$ of $\mathrm{X}$ has a vertex for each $i \in I$. We pass from $M$ to the diagram $M_{J}$ of $l k(\sigma)$ by removing from $M$ all vertices which belong to $I \backslash J[11$, Proposition 3.12].

Since $l k(\sigma)$ is spherical, its diameter (that is $\left.\sup \left\{\mathbf{d}\left(c, c^{\prime}\right): c, c^{\prime} \in \mathscr{C}(l k(\sigma))\right\}\right)$, which we denote by $\operatorname{diam} l k(\sigma)$, is finite. Let us call two chambers $c, c^{\prime}$ opposite if $\mathbf{d}\left(c, c^{\prime}\right)=\operatorname{diam} l k(\sigma)$. As the distance between two chambers in any building is equal to their distance in any apartment containing both, two chambers are opposite if and only if they are opposite in some apartment of $l k(\sigma)$. Moreover two opposite chambers are contained in one and only one apartment, namely conv $\left\{c, c^{\prime}\right\}$.

The following facts are important and useful tools in the applications.

THEOREM 1.1. Let $A$ be an apartment and $c$ a chamber in $A$. Then there exists a unique mapping $\rho_{c, A}: \mathbf{X} \rightarrow A$ such that for all apartments $A^{\prime}$ containing $c$, the map $\left.\rho_{c, A}\right|_{A^{\prime}}$ is the isomorphism fixing $A \cap A^{\prime}$ pointwise.

PROOF. See for example [6, p. 86].

The map $\rho_{c, A}$ is called the retraction of $\mathbf{X}$ onto $A$ with center $c$. Let $\mu$ be the isometry from $A$ to $W$ with $\mu(c)=1$ (the identity element) and define the mapping $\delta_{c}: \mathscr{C} \rightarrow W$ by $\delta_{c}(d)=\mu\left(\rho_{c, A}(d)\right)$. For each $d \in \mathscr{C}$ the group element $\delta_{c}(d)$ retains information about the relative position of $d$ with respect to $c$ : its length is equal to the distance between $c$ and $d$, and the reduced decompositions of $\delta_{c}(d)$ are related to minimal paths from $c$ to $d$. The following proposition shows the properties of the function $\delta_{c}$ and is proved in [12, Section 3].

PROPOSITION 1.2. For any chamber $c$ the following facts hold: 
(i) if $j \in I$, if $d$ and $d^{\prime}$ are two distinct, $j$-adjacent chambers and if $\delta_{c}(d)=w$ then $\delta_{c}\left(d^{\prime}\right) \in\left\{w, w s_{j}\right\}$. Moreover if $l\left(w s_{j}\right)=l(w)+1$ then $\delta_{c}\left(d^{\prime}\right)=w s_{j}$;

(ii) if $\delta_{c}(d)=w$ then for any $j \in I$ there exists a chamber $d^{\prime}$ distinct from $d$ and $j$-adjacent to it such that $\delta_{c}\left(d^{\prime}\right)=w s_{j}$. If $l\left(w s_{j}\right)=l(w)-1$ then there is a unique such $d^{\prime}$.

\section{Measure on the space of apartments}

Let $A_{0}$ be the Coxeter complex of $W$ (that is the representative of the isomorphism class of the set of apartments of $\mathbf{X}$ ). Given any subset of chambers $C \subseteq A_{0}$ we define a map $\lambda: C \rightarrow \mathbf{X}$ to be an isometry if it preserves the labelling and the distance between any two chambers. Denote by $\Lambda$ the set of isometries $\lambda: A_{0} \rightarrow \mathbf{X}$. Note that each apartment in $\mathbf{X}$ is an isometric image $\lambda\left(A_{0}\right)$ of $A_{0}$ into $\mathbf{X}$. Moreover each isometry $\lambda$ is uniquely determined by its image $\lambda\left(A_{0}\right)$ together with its value on a fixed chamber $c_{0}$, because if $\lambda^{\prime}$ is another such isometry, then $\lambda^{-1} \lambda^{\prime}$ is an isometry of $A_{0}$ fixing $c_{0}$ and is therefore the identity of $W$. We endow $\Lambda$ with the open compact topology: a fundamental neighborhood for $\lambda \in \Lambda$ consists of the set of maps $\lambda^{\prime}$ which agree with $\lambda$ on a fixed finite set of chambers. This topology makes $\Lambda$ a locally compact, totally disconnected, Hausdorff and separable space. We remark that the group $W$ acts on $\Lambda$ via $\lambda \mapsto \lambda \circ w$, and that the map $\Lambda \rightarrow \mathscr{A}$ defined by $\lambda \mapsto \lambda\left(A_{0}\right)$, gives a bijective correspondence between the set of $W$-orbits $W \backslash \Lambda$ and $\mathscr{A}$.

Let $c_{0}$ be a fixed chamber in $A_{0}$ and $C_{n}$, for $n \geq 0$, be the compact convex set consisting of the convex hull of $c_{0}$ and the set of chambers which are at distance $n$ from $c_{0},\left(C_{0}=c_{0}\right)$. Let us denote by $\Lambda_{n}$ the set of isometries of $C_{n}$ into $\mathbf{X}$. Let $\psi \in \Lambda_{n}$ and $K_{n, \psi}=\left\{\lambda \in \Lambda:\left.\lambda\right|_{c_{n}}=\psi\right\}$. We remark that the family $\left\{K_{n, \psi}\right\}_{n \in \mathbb{N}, \psi \in \Lambda_{n}}$ forms a basis for the open-compact topology on $\Lambda$. Moreover for each $n$ we get

$$
\Lambda=\bigcup_{\psi \in \Lambda_{n}} K_{n . \psi}
$$

where the union is disjoint.

We denote by $\mathscr{M}$ the $\sigma$-algebra generated by the sets $K_{n, \psi}, n \in \mathbb{N}$ and $\psi \in \Lambda_{n}$ and by $C_{c}(\Lambda)$ the space of continuous, compactly supported functions on $\Lambda$.

PROPOSITION 2.1. If a measure $m$ exists, which is positive, finite on compact sets and regular on $\mathscr{M}$ and such that the following conditions are satisfied

(i) $m\left(K_{0, \psi}\right)=1$,

(ii) for each $n, m\left(K_{n, \psi}\right)$ does not depends on $\psi$,

then it is unique. 
PROOF. Let $m_{1}$ and $m_{2}$ be positive measures satisfying the hypothesis, and let us define on $C_{c}(\Lambda)$ the functional $F_{i}, i=1,2$, by $F_{i}(f)=\int_{\Lambda} f(\lambda) d m_{i}(\lambda)$. We will prove that $F_{1} \equiv F_{2}$. We start by proving that $F_{1} f=F_{2} f$ when $f$ is a finite linear combination of characteristic functions of sets $K_{n, \psi}$. To this end we note that for each $\psi$ and for each $n$ the set $K_{0, \psi}$ can be covered by a finite number of subsets $K_{n, \psi_{1}}, \ldots, K_{n, \psi_{p n}}$. Moreover this covering forms a partition of $K_{0, \psi}$. Since (ii) holds, $p_{n}$ does not depend on $\psi$ and from (i) it follows that $m_{i}\left(K_{n, \psi_{j}}\right)=1 / p_{n}$ for $j=1, \ldots, p_{n}$. As the sets $K_{0, \psi}$ cover $\Lambda$, we can find a finite number of them covering the support of $f$. Without loss of generality we can suppose that the support of $f$ is contained in $K_{0, \psi}$ for some $\psi$. Then there exists $n$, depending on $f$, such that $f=\sum_{j=1}^{p_{n}} b_{i} \chi_{K_{n, \psi_{j}}}$. Then one can easily verify that $F_{1} f=F_{2} f$. Since each continuous compactly supported function is the uniform limit of a sequence of finite linear combination of characteristic functions of sets $K_{n, \psi}$, we conclude that $F_{1}$ and $F_{2}$ define the same functional on $C_{c}(\Lambda)$. Finally the Riesz representation lemma implies that $m_{1}=m_{2}$.

LEMMA 2.2. Let $c$ and $c^{\prime}$ be chambers of $A_{0}$. Each isometry $\psi:\left\{c, c^{\prime}\right\} \rightarrow \mathbf{X}$ extends uniquely to an isometry $\psi^{\prime}: \operatorname{conv}\left(c, c^{\prime}\right) \rightarrow \mathbf{X}$.

Proof. We need to show that for each minimal gallery joining $c$ and $c^{\prime}$ there is a unique minimal gallery of the same (reduced) type joining $\psi^{\prime}(c)$ and $\psi^{\prime}\left(c^{\prime}\right)$. Since $\psi^{\prime}$ send minimal galleries to minimal galleries and is type preserving it is sufficient to prove that if there is a gallery of a given reduced type joining two given chambers this is unique. But this last statement is a well known property of buildings, $[12$, Section 3].

The following Proposition will allow us to prove the existence of $m$. For simplicity in all this section we suppose that our convex sets contain at least one chamber.

PROPOSITION 2.3. Let $C \subseteq C^{\prime}$ be a non-empty, finite and convex subsets of $A_{0}$. Each isometry $\psi: C \rightarrow \mathbf{X}$ extends in a finite number of ways $N\left(C, C^{\prime}\right)$ to an isometry $\psi^{\prime}: C^{\prime} \rightarrow \mathbf{X}$. Moreover $N\left(C, C^{\prime}\right)$ does not depend on $\psi$.

PROOF. By Zorn's lemma it suffices to extend the domain of $\psi$ to a strictly larger convex subset of chambers. We can find a chamber $d \in C$ and $i \in I$ such that $d^{\prime} \notin C$ where $d^{\prime}$ denotes the chamber of $A_{0}$ distinct from and $i$-adjacent to $d$. Let $a$ be any chamber in $\mathbf{X}$ distinct from and $i$-adjacent to $\psi(d)$. We extend $\psi$ by letting $\psi^{\prime}=\psi$ on $C$ and $\psi^{\prime}\left(d^{\prime}\right)=a$. From Lemma 2.2 it follows that $\psi^{\prime}$ is uniquely determined on $C^{\prime}=\operatorname{conv}\left(C, d^{\prime}\right)$. Moreover $N\left(C, C^{\prime}\right)=q_{i}$ and then it does not depend on $\psi$.

We define the measure $m$ by setting

$$
m\left(K_{0, \psi}\right)=1
$$


and since for each isometry $\psi: c_{0} \rightarrow \mathbf{X}$ and for each $n \geq 1$ we have the equality

$$
\left\{\lambda \in \Lambda: \lambda\left(c_{0}\right)=\psi\left(c_{0}\right)\right\}=\bigcup_{\substack{\psi^{\prime} \in \Lambda_{n} \\ \psi^{\prime}\left(c_{0}\right)=\psi\left(c_{0}\right)}} K_{n, \psi^{\prime}}
$$

where the union is disjoint, we set

$$
m\left(K_{n, \psi}\right)=\frac{1}{N\left(C_{0}, C_{n}\right)} .
$$

Let now $C$ be a compact convex set and $\psi: C \rightarrow \mathbf{X}$ be an isometry. We set $K_{C, \psi}=\left\{\lambda \in \Lambda:\left.\lambda\right|_{C}=\psi\right\}$.

COROLlaRY 2.4. The measure $m\left(K_{C, \psi}\right)$ of the compact set $K_{C, \psi}$ does not depend on $\psi$.

PROOF. There exists $n$ such that $C \subseteq C_{n}$. Then the assertion follows from the equality $\left\{\lambda \in \Lambda:\left.\lambda\right|_{C}=\psi\right\}=\bigcup_{\substack{\psi^{\prime} \in \Lambda_{n} \\ \psi^{\prime} \mid C^{\prime}=\psi}} K_{n, \psi^{\prime}}$.

REMARK 2.5. Note that if $c_{0} \in C$ then $m\left(K_{C, \psi}\right)=1 / N\left(c_{0}, C\right)$.

LEMMA 2.6. The measure $m$ is invariant with respect to $W$.

ProOf. Let $w \in W$ and $c=w c_{0}$. Let $C$ be a finite convex set containing $c_{0}$ and $c$ and $\psi: C \rightarrow \mathbf{X}$ be an isometry. We get

$$
m\left(K_{c, \psi}\right)=N(c, C) m\left(K_{C, \psi}\right)=\frac{N(c, C)}{N\left(c_{0}, C\right)} m\left(K_{0, \psi}\right)=\frac{N(c, C)}{N\left(c_{0}, C\right)} .
$$

We will prove that the quotient $N(c, C) / N\left(c_{0}, C\right)$ is equal to 1 . Clearly it does not depend on $C$, so we can choose $C=\operatorname{conv}\left(c_{0}, c\right)$. If $\left(i_{1}, \ldots, i_{\mathrm{d}}\right)$ is the type of a minimal gallery joining $c_{0}$ and $c$ then the number of ways to extend an isometry of $\left\{c_{0}\right\}$ into $\mathbf{X}$ to an isometry of $C$ into $\mathbf{X}$ is equal to the number of galleries of type $\left(i_{1}, \ldots, i_{\mathbf{d}}\right)$ starting at $\psi\left(c_{0}\right)$. This number is equal to $q_{i_{1}} \cdots q_{i_{\mathrm{d}}}$ and is the same as the number of galleries of type $\left(i_{\mathrm{d}}, \ldots, i_{1}\right)$ starting at $\psi(c)$. So condition (i) of Proposition 2.1 holds with $c_{0}$ replaced by $c$. Moreover by Corollary 2.4 it follows that $m$ satisfies condition (ii) of Proposition 2.1 with $C_{n}$ replaced by $w C_{n}$. Then the $W$-invariance of $m$ follows because $m$ is uniquely determined by (i) and (ii) .

\section{LEMMA 2.7. W acts properly on $\Lambda$.}

PROOF. One can easily verify that for every $\lambda \in \Lambda$ the stabilizer $W_{\lambda}$ of $\lambda$ in $W$ is finite (indeed it is trivial) and $\lambda$ has a neighborhood $U$ such that $w U \cap U=\emptyset$ for all $w \in W \backslash W_{\lambda}$. Since $W$ is discrete this is exactly the definition of proper action. 
The fact that $W$ acts properly on $\Lambda$ implies that $W \backslash \Lambda$ is a locally compact, separable, Hausdorff topological space. In the following proposition we state, without proof, a classical result about disintegration of measures known since about 1950 (see for example [5, Section 3]). The formulation given here has been communicated to me by Professor Tim Steger [10].

PROPOSITION 2.8. Let $\Lambda$ be a locally compact, separable, Hausdorff topological space. Let $m$ be a regular and positive measure on $\Lambda$. Suppose that we have a group $W$ which acts properly on $\Lambda$ and preserving $m$. Then there exists a unique regular and positive measure $m_{Q}$ on $W \backslash \Lambda$ such that for each $f \in C_{c}(\Lambda)$ we get

$$
\int_{\lambda \in \Lambda} f(\lambda) d m(\lambda)=\int_{W \lambda \in W \backslash \Lambda}\left(\sum_{w \in W} f(w \lambda)\right) d m_{Q}(W \lambda) .
$$

REMARK 2.9. Let $\pi: \Lambda \rightarrow W \backslash \Lambda$ be the natural projection, and $K \subseteq \Lambda$ be an open compact set such that $\left.\pi\right|_{K}$ is injective. If $f$ is the characteristic function of $K$ then $\sum_{w \in W} f(w \lambda)$ as a function defined on $W \backslash \Lambda$ is the characteristic function of $\pi(K)$. Then equality (2.1) gives

$$
m(K)=m_{Q}(\pi(K))
$$

Let now $C \subset A_{0}$ be a non-empty, finite, convex set, $\psi: C \rightarrow \mathbf{X}$ be an isometry and $K=K_{C, \psi}$. If we identify $W \backslash \Lambda$ with $\mathscr{A}$ we have

$$
\pi(K)=\{A \in \mathscr{A}: \psi(C) \in A\} .
$$

Since $C$ contains at least one chamber the restriction of $\pi$ on $K$ is injective and then

$$
m_{Q}(\{A \in \mathscr{A}: \psi(C) \in A\})=m(K) .
$$

Generally if $C^{\prime}$ is a finite convex set in some apartment of $\mathbf{X}$ we can find a finite convex set $C \subset A_{0}$ and an isometry $\psi: C \rightarrow \mathbf{X}$ such that $\psi(C)=C^{\prime}$. So we can compute the measure of $\mathscr{A}_{C^{\prime}}=\left\{A \in \mathscr{A}: C^{\prime} \in A\right\}$ for each finite convex set $C^{\prime} \subseteq \mathbf{X}$.

REMARK 2.10. For each affine building $\mathbf{X}$, from the definition of $m_{Q}$ it follows immediately that if $c$ is any chamber in $\mathbf{X}$ then $m_{Q}\left(\mathscr{A}_{c}\right)=1$. More generally let $\sigma$ be any simplex in $\mathbf{X}$ and $E$ be any apartment in $l k(\sigma)$. We can identify $\mathscr{C}\left(A_{0}\right)$ with $W$ and then there exists an isometry $\psi$ such that $E=\psi\left(W_{\sigma}\right)$ and $m_{Q}\left(\mathscr{A}_{E}\right)=1 / N\left(1, W_{\sigma}\right)$. If $w_{\sigma}$ denotes the longest element in $W_{\sigma}$, (that is the unique element which is opposite to 1$)$, then $W_{\sigma}=\operatorname{conv}\left(1, w_{\sigma}\right)$. We recall that the reduced expressions for $w_{\sigma}$ are in bijective correspondence with the minimal galleries joining 1 with $w_{\sigma}$. If $s_{i_{1}} \cdots s_{i_{\left\langle w_{\sigma}\right)}}$ is a reduced expression for $w_{\sigma}$ then there exists one (and only one ) gallery of type 
$\left(i_{1}, \ldots, i_{l\left(w_{\sigma}\right)}\right)$ joining 1 with $w_{\sigma}$ and this implies that $N\left(1, W_{\sigma}\right)=q_{i_{1}} \cdots q_{i_{t\left(w_{\sigma}\right)}}$. For each set $\mathscr{E}_{\sigma}^{\prime} \subseteq\left\{A \cap l k(\sigma): A \in \mathscr{A}_{\sigma}\right\}$ let $\mathscr{A}_{\sigma}^{\prime}=\left\{A \in \mathscr{A}_{\sigma}: A \cap l k(\sigma) \in \mathscr{E}_{\sigma}^{\prime}\right\} \subseteq \mathscr{A}_{\sigma}$. As $\mathscr{A}_{\sigma}^{\prime}=\cup_{E \in \mathscr{E}_{\sigma}^{\prime}} \mathscr{A}_{E}$, then, denoting by $|\cdot|$ the cardinality of a given set, we get

$$
m_{Q}\left(\mathscr{A}_{\sigma}^{\prime}\right)=\frac{\left|\mathscr{E}_{\sigma}^{\prime}\right|}{N\left(1, W_{\sigma}\right)} .
$$

Note that if $\sigma$ has corank two and $l k(\sigma)$ has parameters $\left\{q_{s}, q_{t}\right\}$ then $N\left(1, W_{\sigma}\right)$ is equal to $q_{s}^{\left(l\left(w_{\sigma}\right)+1\right) / 2} q_{t}^{\left(l\left(w_{\sigma}\right)-1\right) / 2}$ if $l\left(w_{\sigma}\right)$ is odd and to $\left(q_{s} q_{t}\right)^{l\left(w_{\sigma}\right) / 2}$ if $l\left(w_{\sigma}\right)$ is even. Indeed, if ...stst is the longest word in $W_{\sigma}$ this follows because there exists exactly one gallery of type $(\ldots, s, t, s, t)$ joining any two opposite chambers. Note also that, as there exists one (and only one) of type $(\ldots, t, s, t, s)$ joining the same chambers, then if $l\left(w_{\sigma}\right)$ is odd, exchanging the role of $q_{s}$ and $q_{t}$ we have that $q_{s}=q_{t}$ (that is a rank-two spherical building is necessarily homogeneous if its diameter is odd).

\section{Inversion of the Radon transform}

We define a strip $\mathscr{S}$ in a given apartment as the intersection $\alpha \cap \alpha^{\prime}$ of two halfapartments $\alpha$ and $\alpha^{\prime}$ such that $\alpha$ contains the opposite of $\alpha^{\prime}$.

Let $\mathscr{S}$ be a strip and let $\partial \alpha \cup \partial \alpha^{\prime}$ its boundary. Each wall $l$ not parallel to $\partial \alpha$ (equivalently to $\partial \alpha^{\prime}$ ) splits $\mathscr{S}$ in two subsets $B$ and $B^{\prime}$ such that $\mathscr{C}(B) \cap \mathscr{C}\left(B^{\prime}\right)=\emptyset$ and $\mathscr{C}(B) \cup \mathscr{C}\left(B^{\prime}\right)=\mathscr{C}(\mathscr{S})$. We say that $B$ and $B^{\prime}$ are the opposite half-strips originating from $\partial l \cap \mathscr{S}$. Let $B$ and $B^{\prime}$ be opposite half-strips and denote by $B B^{\prime}$ the strip $\mathscr{S}$ such that $\mathscr{S}=B \cup B^{\prime}$. Similar conventions will hold for unions of half-apartments and so on

If $g$ is a complex-valued function defined on $\mathscr{C}$ set $\Psi g(\mathscr{S})=\sum_{c \in \mathscr{S}} g(c)$ for each strip $\mathscr{S}$, whenever the right-hand side is meaningful.

LEMMA 3.1. Let $\mathscr{S}$ be a strip in $\mathbf{X}$. Then there exist half-apartments $\alpha, \beta, \gamma, \delta$, with interiors disjoint from $\mathscr{S}$ and from each other, such that $\alpha \mathscr{S} \gamma, \beta \mathscr{S} \delta, \alpha \beta, \gamma \delta$ are apartments; if $g \in L^{1}(\mathscr{C}(A))$ for each apartment $A \in \mathscr{A}$, then

$$
2 \Psi g(\mathscr{S})=R g(\alpha \mathscr{S} \gamma)+R g(\beta \mathscr{S} \delta)-R g f(\alpha \beta)-R g(\gamma \delta) .
$$

PROOF. Let $\mathscr{S}=\alpha^{\prime} \cap \alpha^{\prime \prime}$. We can take for $\alpha$ the opposite of $\alpha^{\prime}$ and for $\delta$ the opposite of $\alpha^{\prime \prime}$. Then the existence of $\beta$ and $\gamma$ is guaranteed by the hypothesis that $\mathbf{X}$ is thick. We have $R g(\alpha \mathscr{S} \gamma)=\sum_{c \in \alpha} g(c)+\sum_{c \in \mathscr{S}} g(c)+\sum_{c \in \beta} g(c)$, and so forth, so the right-hand side of (3.1) yields $2 \sum_{c \in \mathscr{S}} g(c)$ which is equal to the left-hand side.

Case I: $\mathbf{X}$ with diagram of type $\widetilde{A_{2}}$ 
LEMMA 3.2. Let $c$ be any chamber in $\mathbf{X}$ and denote by $\sigma_{i}, i=0,1,2$, its edges. Then there exist half-strips $B_{i j}, i, j=0,1,2$ and $j \neq i$ with interiors disjoint from $c$ and disjoint from each other, such that $B_{i j}$ originates from $\sigma_{i}$ and $B_{i j} c$ is a strip originating from $\sigma_{j}$ and such that $B_{i j} c B_{j i}$ and $B_{i j_{1}} B_{i j_{2}}$, for $j_{1} \neq j_{2}$ are strips. If $g \in L^{1}(\mathscr{C}(A))$ for each $A \in \mathscr{A}$ then

$$
3 g(c)=\sum_{i<j} \Psi g\left(B_{i j} c B_{j i}\right)-\sum_{i \neq j_{1} \neq j_{2}} \Psi g\left(B_{i j_{1}} B_{i j_{2}}\right)
$$

PROOF. We can suppose that $\sigma_{i}$ has cotype $i$. Let $A$ be any apartment containing $c$ and let $\partial \Phi_{i}$ be the wall in $A$ corresponding to $\sigma_{i}$. Let $x_{i}$ be the vertex of $c$ of type $i$ and note that the subgroup of $W$ which stabilizes $x_{i}$ in $A$ is generated by $s_{i^{+}}$and $s_{i^{-}}$where $i^{ \pm}=i \pm 1 \bmod 3$. Since the element $s_{i} s_{i}+s_{i^{-}} s_{i^{+}}$has infinite order then $\partial \Phi_{i^{+}+i^{+}}$is the (unique) wall in $A$ containing $x_{i}$ and parallel to $\partial \Phi_{i}$. Consider the strips $\mathscr{S}_{i}=\Phi_{i}^{+} \cap \Phi_{i^{+} i^{-} i^{+}}^{+}$and let $B_{i^{+} i^{-}}^{\prime}=\mathscr{S}_{i} \cap \Phi_{i^{+}}^{-}$and $B_{i^{-i^{+}}}=\mathscr{S}_{i} \cap \Phi_{i^{-}}^{-}$and note that since $c=\bigcap_{i \in I} \Phi_{i}^{+}$then $\mathscr{S}_{i}=B_{i^{+} i^{-}}^{\prime} c B_{i^{-} i^{+}}$. Let $\Pi_{i^{+}}$be any half-apartment in $\mathbf{X}$ whose interior is disjoint from $\Phi_{i^{+}}^{+}$and $\Phi_{i^{+}}^{-}$and such that $\partial \Pi_{i^{+}}=\partial \Phi_{i^{+}}$. Hence $\Pi_{i^{+}} \cup \Phi_{i^{+}}^{+}$ and $\Pi_{i^{+}} \cup \Phi_{i^{+}}^{-}$are apartments.

Consider the map $\left.\rho_{c, A}\right|_{\Pi_{i^{+}} \cup \Phi_{i^{+}}^{+}}: \Pi_{i^{+}} \cup \Phi_{i^{+}}^{+} \rightarrow A$. It is the unique isomorphism of $\Pi_{i^{+}} \cup \Phi_{i^{+}}^{+}$onto $A$ fixing $c B_{i^{-i^{+}}}$. Denote by $B_{i^{+} i^{-}}$the half-strip in $\Pi_{i^{+}}$such that its image under $\rho_{c, A}$ is the half-strip $B_{i^{+} i^{-}}^{\prime}$. Then $B_{i^{+} i^{-}} c B_{i^{-} i^{+}}$is a strip in $\Pi_{i^{+}} \cup \Phi_{i^{+}}^{+}$ isomorphic and isometric to $\mathscr{S}_{i}$ which we denote by $\mathscr{S}_{i}^{\prime}$.

Let $j=i^{-}$and note that the reflection $s_{i^{+}}$maps $B_{i^{+} j^{-}}^{\prime}$ onto $\mathscr{S}_{j} \cap \Phi_{j^{-}}^{+}=B_{j^{+}-}^{\prime} c$. Consider the isomorphism of $\Pi_{i^{+}} \cup \Phi_{i^{+}}^{-}$onto $A$ which is equal to the identity on $\Phi_{i^{+}}^{-}$and is equal to $s_{i^{+}} \circ \rho_{c, A}$ on $\Pi_{i^{+}}$. It maps $B_{i^{+i^{-}}} B_{j^{-j^{+}}}$isometrically onto $\mathscr{S}_{j}$. Then $B_{i^{+} i^{-}} B_{j-j^{+}}$is a strip in $\Pi_{i^{+}} \cup \Phi_{i^{+}}^{-}$whose interior is disjoint from $c$ and which we denote by $\mathscr{S}_{i}^{\prime \prime}$. Considering the strips $\mathscr{S}_{i}^{\prime}$ and $\mathscr{S}_{i}^{\prime \prime}, i=0,1,2$, as we have that $\Psi g\left(\mathscr{S}_{i}^{\prime}\right)=\sum_{d \in B_{i^{+} i^{-}}} g(d)+g(c)+\sum_{d \in B_{i^{-} i^{+}}} g(d)$, and so forth, we get that the right hand side of (3.2) is equal to the left hand-side and this proves the assertion. (In Figure 2 the half-strips $B_{i^{+} i^{-}}$are dashed to mean that they do not lie in the same apartment containing the half-strips $B_{i^{-} i^{+}}$.)

Let $a(c)$ be the right-hand side of (3.2). Then, for each $c \in \mathscr{C}$, Lemma 3.1 allows an expression of $a(c)$ and then of $g(c)$ in terms of $R g$. So $g$ is uniquely determined by $R g$.

\section{Case II: $\mathbf{X}$ with diagram of type $\widetilde{C}_{2}$}

Let $c$ be any chamber in $\mathbf{X}$ and let $x_{0}$ be its vertex with the property that $l k\left(x_{0}\right)$ is isomorphic to a spherical building with diagram of type $A_{1} \times A_{1}$. Suppose that $x_{0}$ has type 0 , and denote by $\sigma_{0}$ the edge of $c$ opposite to $x_{0}$.

LEMMA 3.3. Let $c^{\prime}$ be a chamber distinct from $c$ and 0 -adjacent to it. Let $\sigma_{1}$ and 


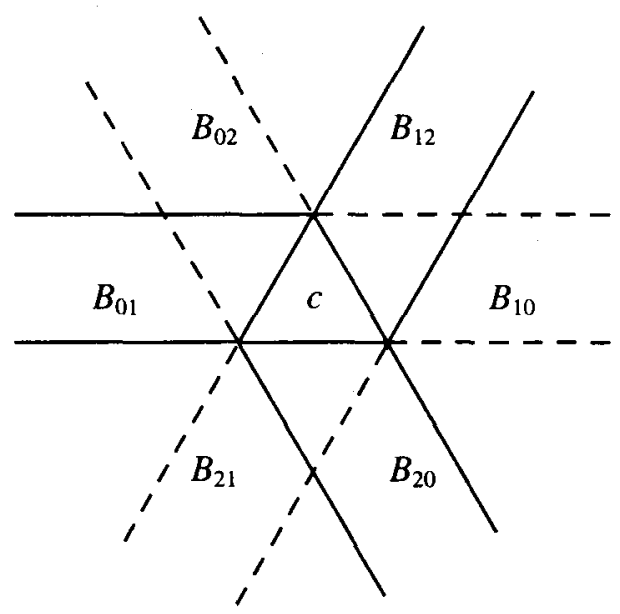

FIGURE 2.

$\sigma_{2}$, (respectively $\sigma_{1}^{\prime}$ and $\left.\sigma_{2}^{\prime}\right)$, be the edges of $c$ (respectively $\left.c^{\prime}\right)$ of cotype 1 and 2 . Then there exist half-strips $B_{1}, \widetilde{B}_{1}, B_{2}, \widetilde{B}_{2}$, with interiors disjoint from $c, c^{\prime}$ and from each other, such that $B_{1}, \widetilde{B}_{1}$ originate from $\sigma_{1}$ and $B_{2}, \widetilde{B}_{2}$ originate from $\sigma_{2}^{\prime}$, and such that $B_{1} c c^{\prime} B_{2}, \widetilde{B}_{1} c c^{\prime} \widetilde{B}_{2}, B_{1} \widetilde{B}_{1}, B_{2} \widetilde{B}_{2}$ are strips; if $g \in L^{1}(\mathscr{C}(A))$ for each $A \in \mathscr{A}$ then

$$
2\left(g(c)+g\left(c^{\prime}\right)\right)=\Psi g\left(B_{1} c c^{\prime} B_{2}\right)+\Psi g\left(\widetilde{B_{1}} c c^{\prime} \widetilde{B}_{2}\right)-\Psi g\left(B_{1} \widetilde{B}_{1}\right)-\Psi g\left(B_{2} \widetilde{B}_{2}\right) .
$$

PROOF. We can argue as in the proof of Lemma 3.2. The details are left to the interested reader.

Denote by $a\left(c, c^{\prime}\right)$ the right-hand side of (3.3) and let $c^{\prime \prime}$. be a chamber 0-adjacent to $c$ and different from $c$ and $c^{\prime}$. Lemma 3.3 applied to $c, c^{\prime \prime}$ and then to $c^{\prime}, c^{\prime \prime}$ allows an expression of $g(c)$ in terms of $\Psi$. Indeed

$$
4 g(c)=a\left(c, c^{\prime}\right)+a\left(c, c^{\prime \prime}\right)-a\left(c^{\prime}, c^{\prime \prime}\right) .
$$

Finally, for each $c \in \mathscr{C}$, Lemma 3.1 allows us to express the right hand side of (3.4) by a finite linear combination of values of $R g$.

Case III: $\mathbf{X}$ with diagram of type $\widetilde{G_{2}}$.

Label the vertices of $\mathbf{X}$ in such a way that the type 0 (respectively 1,2 ) corresponds to the vertices $v$ such that $l k(v)$ has diagram of type $A_{2}$ (respectively $l k(v)$ has diagram of type $A_{1} \times A_{1}, l k(v)$ has diagram of type $\left.G_{2}\right)$. Note that the affine Coxeter group $W\left(\widetilde{A_{2}}\right)$ of type $\widetilde{A_{2}}$ is a subgroup (of index six) of the affine Coxeter group $W\left(\widetilde{G_{2}}\right)$ of 
type $\widetilde{G}_{2}$ associated to $\mathbf{X}$. So the set of walls, and then the set of half-apartments of any apartment of $\mathbf{X}$ contains the set of walls, (respectively the set of half-apartments) of the complex associated to $W\left(\widetilde{A_{2}}\right)$. (From the tiling induced by $W\left(\widetilde{G_{2}}\right)$ we can recover the tiling induced by $W\left(\widetilde{A_{2}}\right)$ considering pairs of distinct 0 -adjacent chambers.)

Let $c$ and $c^{\prime}$ be any two distinct 0 -adjacent chambers and let $\sigma_{1}$ and $\sigma_{2}$ (respectively $\sigma_{1}{ }^{\prime}$ and $\sigma_{2}{ }^{\prime}$ ) the edges of $c$ (respectively $c^{\prime}$ ) of cotype 1 and 2 . Set $l_{1}=\sigma_{1}, l_{2}=\sigma_{1}^{\prime}$ and $l_{3}=\sigma_{2} \cup \sigma_{2}{ }^{\prime}$, then arguing as in the case of a building of type $\widetilde{A_{2}}$ we get the following lemma.

LEMMA 3.4. Define $c$ and $c^{\prime}$ as above. Then there exist half-strips $B_{i j}, i, j=1,2,3$ and $j \neq i$ with interiors disjoint from $c, c^{\prime}$ and from each other, such that $B_{i j}$ originates from $l_{i}$ and $B_{i j} c c^{\prime}$ is a strip originating from $l_{j}$ and such that $B_{i j} c c^{\prime} B_{j i}$ and $B_{i j_{1}} B_{i j_{2}}$ are strips: if $g \in L^{1}(\mathscr{C}(A))$ for each $A \in \mathscr{A}$ then

$$
g(c)+g\left(c^{\prime}\right)=\frac{1}{3}\left(\sum_{i<j} \Psi g\left(B_{i j} c c^{\prime} B_{j i}\right)-\sum_{i \neq j_{1} \neq j_{2}} \Psi g\left(B_{i j_{1}} B_{i j_{2}}\right)\right) .
$$

Consider now another distinct chamber $c^{\prime \prime} 0$-adjacent to $c$. Then Lemma 3.4 applied to $c, c^{\prime \prime}$ and then to $c^{\prime}, c^{\prime \prime}$ allows an expression of $g(c)+g\left(c^{\prime \prime}\right)$ and of $g\left(c^{\prime}\right)+g\left(c^{\prime \prime}\right)$ in terms of $\Psi$. Proceeding as in the case of a building of type $\widetilde{C}_{2}$ we can recover the value of $g$ in $c$ in terms of $R g$.

We have just proved the following proposition.

PROPOSITION 3.5. Let $\mathbf{X}$ be any rank three affine building. The operator $R$ is injective on the space of functions that are summable on the set of chambers of each apartment of $\mathbf{X}$.

\section{Inversion formulas}

From now on, given any chamber $c$ in $\mathbf{X}$ we will denote by $A_{0}$ a fixed apartment containing $c$ and by $\rho$ the retraction of $\mathbf{X}$ on $A_{0}$ with center $c$. Given a chamber $d$ and a simplex $\sigma$ of $\mathbf{X}$ we will denote by $\operatorname{proj}_{\sigma} d$ (projection of $d$ on $\sigma$ ) the unique chamber of $l k(\sigma)$ nearest to $d$, (that is, the first element of any stretched gallery from $\sigma$ to $d$ ), $[9$, Corollary 3.9].

Proposition 4.1. Let $\sigma \subset c$ and $A \in \mathscr{A}_{\sigma}$. Then

$$
\mathscr{C}(\rho(A))=\bigcup_{d \in \mathscr{B}(A) \cap l k(\sigma)} \operatorname{proj}_{\sigma}^{-1}(\rho(d)) \cap \mathscr{C}\left(A_{0}\right) .
$$


Proof. For each chamber $d \in A \cap l k(\sigma)$ let $L_{d}$ be the convex subcomplex $\operatorname{proj}_{\sigma}^{-1}(d) \cap \mathscr{C}(A)([11$, Proposition 2.32]). Note that

$$
\mathscr{C}(A)=\bigcup_{d \in A \cap l k(\sigma)} L_{d}
$$

where the union is disjoint. We will show that

$$
\rho\left(L_{d}\right)=\operatorname{proj}_{\sigma}^{-1} \rho(d) \cap \mathscr{C}\left(A_{0}\right) .
$$

From this our assertion will follow. Note that, as the retraction $\rho$ maps every stretched gallery starting from $\sigma$, onto a stretched gallery with same origin, one has that

$$
\rho\left(L_{d}\right) \subseteq \operatorname{proj}_{\sigma}^{-1} \rho(d) \cap \mathscr{C}\left(A_{0}\right) .
$$

To prove that equality holds we argue as follows. Letting $\rho_{d . A}$ the retraction on $A$ with center $d$, then the set $L_{d} \cup\{c\}$ is isometric to $L_{d} \cup\left\{\rho_{d, A}(c)\right\} \subset A$ and this implies ([9, Theorem 3.6]) that there exists an apartment $A^{\prime}$ containing $L_{d}$ and $c$. Let $\varphi: A^{\prime} \rightarrow A_{0}$ be the unique isomorphism fixing $A^{\prime} \cap A_{0}$; then the image of $L_{d}$ under $\varphi$ is equal to $\operatorname{proj}_{\sigma}^{-1} \varphi(d) \cap \mathscr{C}\left(A_{0}\right)$. Therefore, as $\varphi$ and $\rho$ agree pointwise on $L_{d}$, we have proved the proposition.

Let $A$ be any apartment in $\mathbf{X}$. As $A$ is the collection of its chambers, the 'position' of $A$ with respect to $c$ is completely known if for each chamber $d \in A$ we know the value at $d$ of the function $\delta_{c}$ or, equivalently, if we look at the image of $A$ under $\rho$. In view of [11, Proposition 2.32], the content of Proposition 4.1 is that to know the image of $A$ under $\rho$ it is sufficient to know the image of $A \cap l k(\sigma)$ under the restriction of $\rho$ to $l k(\sigma)$.

If $\Phi \subseteq A_{0}$ is a convex chamber subcomplex, we set

$$
\mathscr{A}^{\Phi}=\{A \in \mathscr{A}: \rho(A)=\Phi\} .
$$

In particular $\Phi$ can be $\Phi_{r}^{+}$or $\Phi_{r}^{-}$for some reflection $r$ in $W$. In the sequel, to simplify the notation, we will write $\Phi_{r}$ for $\Phi_{r}^{-}$.

We will radialize with respect to $m_{Q}$ the discrete inversion formulas of Section 3 . To this end it will be necessary to derive case by case the measure of suitable subsets of $\mathscr{A}$. We now prove some technical lemmas.

Lemma 4.2. Let $(\mathbf{X}, \mathscr{A})$ be a spherical building with diagram of type $A_{1}$, with $S=\{s\}$ and parameter $q_{s}$. Then

$$
\left|\mathscr{A}^{\Phi_{s}}\right|=\frac{q_{s}\left(q_{s}-1\right)}{2}
$$


PROOF. As $\mathbf{X}$ is a rank-one building, each apartment consists of a pair of distinct, $s$-adjacent chambers. Moreover $\Phi_{s}=\{s\}$. Then the requested number of apartments is equal to the number of unordered pairs $\left(d_{0}, d_{1}\right)$ of distinct chambers such that $\delta_{c}\left(d_{0}\right)=\delta_{c}\left(d_{1}\right)=s$, and this number turns out be equal the right-hand side of (4.1).

REMARK 4.3. Let $\mathbf{X}$ be any building. Denote by $\Gamma\left(w_{0}, \ldots, w_{n}\right)=\Gamma_{i_{1}, \ldots, i_{n}}\left(w_{0}, \ldots\right.$, $\left.w_{n}\right), w_{k} \in W$ for $0 \leq k \leq n$, the collection of simple galleries $\left(d_{0}, \ldots, d_{n}\right)$ of reduced type $\left(i_{1}, \ldots, i_{n}\right)$, such that $\delta_{c}\left(d_{k}\right)=w_{k}$ for all $0 \leq k \leq n$. For each $d \in \mathscr{C}$ such that $\delta_{c}(d)=w_{k}$, denote by $a\left(w_{k}, i_{k+1}, w_{k+1}\right)$ the number of distinct chambers $d^{\prime}$ $i_{k+1}$-adjacent to $d$ such that $\delta_{c}\left(d^{\prime}\right)=w_{k+1}$. Then an easy induction shows that

$$
\left|\Gamma\left(w_{0}, \ldots, w_{n}\right)\right|=\phi\left|\delta_{c}^{-1}\left(w_{0}\right)\right| \prod_{0 \leq k \leq n-1} a\left(w_{k}, i_{k+1}, w_{k+1}\right)
$$

where

$$
\phi= \begin{cases}\frac{1}{2} & \text { if } n \text { is odd, } w_{j}=w_{n-j} \text { for all } j \text { and }\left(i_{1}, \ldots, i_{n}\right)=\left(i_{n}, \ldots, i_{1}\right) \\ 1 & \text { otherwise. }\end{cases}
$$

Recall that $w_{k+1} \in\left\{w_{k}, w_{k} s_{i_{k}}\right\}$ and note that from Proposition 1.2 it follows immediately that

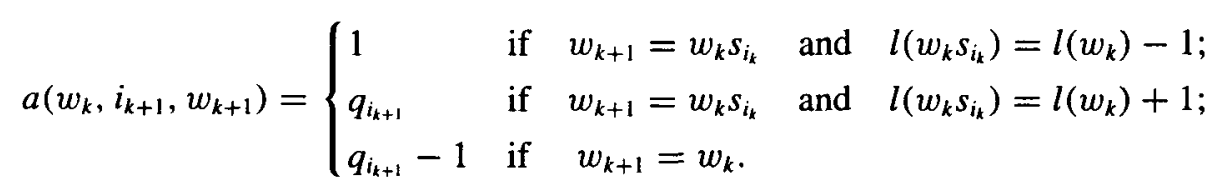

REMARK 4.4. Note that if $w=s_{i_{1}} \cdots s_{i_{i}}$ is a reduced expression for $w \in W$ then $\left|\delta_{c}^{-1}(w)\right|$ is the same as the number of minimal galleries of type $\left(i_{1}, \ldots, i_{l}\right)$ starting at c.

LEMMA 4.5. Let $(\mathbf{X}, \mathscr{A})$ be a spherical building with diagram of type $A_{2}$, with $S=\{s, t\}$ and parameter $q$. Then the sets $\mathscr{A}^{\Phi_{s t s}}$, (note that sts $\left.=t s t\right), \mathscr{A}^{\Phi_{s s}} \cap \Phi_{s}$ and $\mathscr{A}^{\Phi_{s t s} \cap \Phi_{1}}$ are not empty and the following equalities hold:

$$
\begin{aligned}
\left|\mathscr{A}^{\Phi_{s i s}}\right| & =\frac{(q-1) q^{3}}{2} \\
\left|\mathscr{A}^{\Phi_{s} \cap \Phi_{s s s}}\right| & =\left|\mathscr{A}^{\Phi, \cap \Phi_{s t s}}\right|=\frac{(q-1)^{2} q^{3}}{2} .
\end{aligned}
$$


PROOF. Let $\left(d_{0}, \ldots, d_{n}\right)$ any simple gallery of reduced type and length $n=\operatorname{diam} \mathbf{X}$ and denote by $\gamma^{\prime}\left(d_{0}, d_{n}\right)=\left(d_{0}, d_{1}^{\prime}, \ldots, d_{n-1}^{\prime}, d_{n}\right)$ the unique gallery which exhausts conv $\left\{d_{0}, d_{n}\right\}$. Starting with a chamber $d_{0}$ such that $\delta_{c}\left(d_{0}\right) \in \Phi$ we first construct all galleries $\left(d_{0}, \ldots, d_{n}\right)$, (for $\left.n=3\right)$, such that $\delta_{c}\left(d_{i}\right) \in \Phi, 1 \leq i \leq n$, then we look at the image under $\delta_{c}$ of conv $\left\{d_{0}, d_{n}\right\}$.

Let $\Phi=\Phi_{s t s}$ and choose $d_{0}$ such that $\delta_{c}\left(d_{0}\right)=s t$. Then as any apartment in $\mathbf{X}$ contains exactly one gallery of type $(s, t, s)$ and exactly one gallery of type $(t, s, t)$, we can suppose, without loss of generality, that $\left(d_{0}, \ldots, d_{3}\right)$ has type $(t, s, t)$. Then starting from $d_{0}$ and applying Proposition 1.2 repeatedly, we see that for such a gallery to have its term $d_{i}$ such that $\delta_{c}\left(d_{i}\right) \in \Phi_{s t s}=\{s t, s t s, t s\}, 1 \leq i \leq 3$, only the following two possibilities can occur: either $\left(d_{0}, \ldots, d_{3}\right) \in \Gamma(s t, s t, s t s, t s)$, or $\left(d_{0}, \ldots, d_{3}\right) \in \Gamma(s t, s t, s t s, s t s)$. If $\left(d_{0}, \ldots, d_{3}\right) \in \Gamma(s t, s t, s t s, t s)$ then, being $d_{1}^{\prime} \sim d_{0}$ and $l\left(\delta_{c}\left(d_{0}\right) s\right)=l\left(\delta_{c}\left(d_{0}\right)\right)+1$, we have that $\delta_{c}\left(d_{1}^{\prime}\right)=s t s$. While, being $d_{2}^{\prime} \sim d_{3}$ and $l\left(\delta_{c}\left(d_{3}\right) s\right)=l\left(\delta_{c}\left(d_{3}\right)\right)-1$ we get $\delta_{c}\left(d_{2}^{\prime}\right)=t$ or $\delta_{c}\left(d_{2}^{\prime}\right)=\delta_{c}\left(d_{3}\right)$. In the first case it cannot be $d_{1}^{\prime} \sim d_{2}^{\prime}$. Then necessarily $\delta_{c}\left(d_{2}^{\prime}\right)=t s$. Note that in this case $\delta_{c}$ maps conv $\left\{d_{0}, d_{3}\right\}$ onto $\Phi_{s t s}$ (see Figure 3(a)). Analogously if $\left(d_{0}, \ldots, d_{3}\right) \in$ $\Gamma(s t, s t, s t s, s t s)$ one can prove that necessarily $\delta_{c}\left(d_{1}^{\prime}\right)=\delta_{c}\left(d_{2}^{\prime}\right)=s t s$ and in this case $\delta_{c}$ maps conv $\left\{d_{0}, d_{3}\right\}$ onto $\{s t, s t s\}=\Phi_{s} \cap \Phi_{s t s}$. (see Figure 3(b))

As each apartment in $\mathscr{A}^{\Phi_{s t s}}$ and in $\mathscr{A}^{\Phi_{s t s} \cap \Phi_{s}}$ contains a chamber $d_{0}$ such that $\delta_{c}\left(d_{0}\right)=$ $s t$ in this way we can construct all apartments in $\mathscr{A}^{\Phi_{s s s}}$ and in $\mathscr{A}^{\Phi_{s s} \cap \Phi_{s}}$. Note that the correspondence between $\Gamma_{t, s, t}(s t, s t, s t s, t s)$ (respectively $\Gamma_{t, s, t}(s t, s t, s t s, s t s)$ ) and $\mathscr{A}^{\Phi_{s t s}}$ (respectively $\mathscr{A}^{\Phi_{s s} \cap \Phi_{s}}$ ) is 2 -1. In fact each apartment in $\mathscr{A}^{\Phi_{s t s}}$ (respectively in $\mathscr{A}^{\Phi_{s t s} \cap \Phi_{s}}$ ) contains two distinct galleries in $\Gamma_{t, s, t}(s t, s t, s t s, t s)$ (respectively in $\left.\Gamma_{t, s, t}(s t, s t, s t s, s t s)\right)$ : indeed $\left(d_{0}, d_{1}, d_{2}, d_{3}\right)$ and $\left(d_{1}, d_{0}, d_{1}^{\prime}, d_{2}^{\prime}\right)$. Therefore by Remark 4.3 we get (4.2) and part of (4.3).

Let now $d_{0}$ be such that $\delta_{c}\left(d_{0}\right)=t s$, and suppose that $\gamma\left(d_{0}, d_{3}\right)$ has type $(s, t, s)$. This case can be considered as being symmetric to the preceding case, so we obtain again apartments in $\mathscr{A}^{\Phi_{s t s}}$ by choosing $d_{1}$ such that $\delta_{c}\left(d_{1}\right)=t s$ and $d_{3}$ such that $\delta_{c}\left(d_{3}\right)=s t$. We obtain apartments in $\mathscr{A}^{\Phi_{s t s} \cap \Phi_{t}}$ by choosing $d_{1}$ as above and $d_{3}$ such that $\delta_{c}\left(d_{2}\right)=\delta_{c}\left(d_{3}\right)=s t s$. This completes the proof of (4.3).

LEMMA 4.6. Let $(\mathbf{X}, \mathscr{A})$ be a spherical building with diagram of type $B_{2}$, with $S=\{s, t\}$ and parameters $q_{s}, q_{t}$. Then the sets $\mathscr{A}^{\Phi_{s t s}}$ and $\mathscr{A}^{\Phi_{s t} \cap \Phi_{t}}$ are not empty and we have

$$
\left|\mathscr{A}^{\Phi_{s i s}}\right|=\left(q_{s} q_{t}\right)^{2} \frac{\left(q_{t}-1\right)}{2} .
$$

If $q_{t}>2$ then the set $\mathscr{A}^{\Phi_{s t s} \cap \Phi_{r}}$ is the union of two disjoint subset $\left(\mathscr{A}^{\Phi_{s s} \cap \Phi_{t}}\right)^{\prime}$ and 


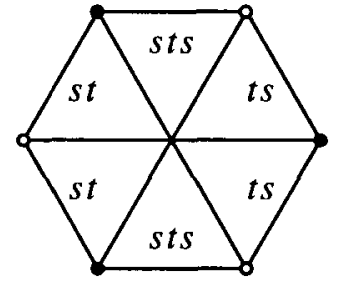

(a)

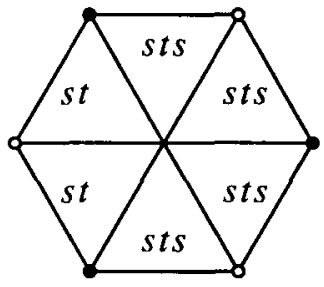

(b)

FIGURE 3.

$\left(\mathscr{A}^{\Phi_{s s} \cap \Phi_{t}}\right)^{\prime \prime}$ such that

$$
\begin{aligned}
& \left|\left(\mathscr{A}^{\Phi_{s t} \cap \Phi_{t}}\right)^{\prime}\right|=\left(q_{s} q_{t}\right)^{2} \frac{\left(q_{t}-1\right)^{2}}{4}, \\
& \left|\left(\mathscr{A}^{\Phi_{s s} \cap \Phi_{t}}\right)^{\prime \prime}\right|=\left(q_{s} q_{t}\right)^{2} \frac{\left(q_{s}-1\right)\left(q_{t}-1\right)\left(q_{t}-2\right)}{2} .
\end{aligned}
$$

The same formulas hold exchanging the roles of $s$ and $t$.

PROOF. We argue as in the proof of Lemma 4.5 and use the same notation: starting from any $w \in W$ (the dihedral group of order eight), such that $l(s t s w)<l(w)$ (respectively $l(s t s w)<l(w)$ and $l(t w)<l(w))$ we first construct all simple galleries $\left(d_{0}, \ldots, d_{n}\right)$, for $n=4$, such that $\delta_{c}\left(d_{0}\right)=w$ and that for $0 \leq i \leq 4, \delta_{c}\left(d_{i}\right)$ satisfies the same inequality or inequalities.

Let $w=t$ st (this choice is convenient as $w \in \Phi_{s t s} \cap \Phi_{t}$ ), and suppose that $\left(d_{0}, \ldots, d_{4}\right)$ has type $(t, s, t, s)$. Then applying at each step Proposition 1.2 and denoting by $\underline{w}$ the longest element stst $=t s t s$ in $W$, one can verify that only the following four cases occur:

(1) $\left(d_{0}, \ldots, d_{4}\right) \in \Gamma_{t, s, t . s}(t s t, t s t, \underline{w}, s t s, s t)$

(2) $\left(d_{0}, \ldots, d_{4}\right) \in \Gamma_{t, s, t, s}(t s t, t s t, \underline{w}, \underline{w}, t s t)$

(3) $\left(d_{0}, \ldots, d_{4}\right) \in \Gamma_{t, s, t, s}(t s t, t s t, \underline{w}$, sts, sts)

(4) $\left(d_{0}, \ldots, d_{4}\right) \in \Gamma_{t, s, t, s}($ tst, tst $, \underline{w}, \underline{w}, \underline{w})$

and that, respectively, for $\gamma^{\prime}\left(d_{0}, d_{4}\right)$ we get:

(1') $\gamma^{\prime}\left(d_{0}, d_{4}\right) \in \Gamma_{s, t, s, t}(t s t, \underline{w}, s t s, s t, s t)$

(2') $\gamma^{\prime}\left(d_{0}, d_{4}\right) \in \Gamma_{s, t, s, t}(t s t, \underline{w}, \underline{w}, t s t, t s t)$

(3') $\gamma^{\prime}\left(d_{0}, d_{4}\right) \in \Gamma_{s, t, s, t}(t s t, \underline{w}, \underline{w}, \underline{w}, s t s)$

(4') either $\gamma^{\prime}\left(d_{0}, d_{4}\right) \in \Gamma_{s . t . s, t}(t s t, \underline{w}$, sts, sts, $\underline{w})$ or $\gamma^{\prime}\left(d_{0}, d_{4}\right) \in \Gamma_{s, t, s, t}(t s t, \underline{w}, \underline{w}, \underline{w}$, w).

As $\Phi_{s t s}=\{t s t, \underline{w}, s t s, s t\}$, only case (1) leads to apartments in $\mathscr{A}^{\Phi_{s t s}}$, and (4.4) follows as the correspondence between this set and the set of galleries as in (1) is a 
1-2 correspondence.

If $\left(d_{0}, \ldots, d_{4}\right)$ is as in (2) then $\delta_{c}$ maps conv $\left\{d_{0}, d_{4}\right\}$ onto $\{t s t, \underline{w}\}=\Phi_{t} \cap \Phi_{s t s}$. Denote by $\left(\mathscr{A}^{\Phi_{s s} \cap \Phi_{t}}\right)^{\prime}$ the set of apartments like conv $\left\{d_{0}, d_{4}\right\}$ and note that there is a 4-1 correspondence between $\Gamma_{t, s, t, s}(t s t, t s t, \underline{w}, \underline{w}, t s t)$ and $\left(\mathscr{A}^{\Phi_{s t s} \cap \Phi_{t}}\right)^{\prime}$. From this we obtain (4.5).

Suppose now that $\left(d_{0}, \ldots, d_{4}\right)$ is as in (3). In this case $\delta_{c}$ maps conv $\left\{d_{0}, d_{4}\right\}$ onto $\{t s t, \underline{w}, s t s\}=\Phi_{s t s} \cap \Phi_{t s t}$. If we denote by $\left(\mathscr{A}^{\Phi_{s t s} \cap \Phi_{t s t}}\right)^{\prime}$ the set of apartments that we obtain from such galleries, then we get $\mid\left(\mathscr{A}^{\left.\Phi_{s t s} \cap \Phi_{t s t}\right)^{\prime}}|=| \Gamma_{t, s, t, s}(t s t, t s t, \underline{w}\right.$, sts, sts $) \mid$, as each apartment in $\left(\mathscr{A}^{\Phi_{s s t} \cap \Phi_{t s t}}\right)^{\prime}$ contains only one gallery in $\Gamma_{t, s, t, s}(t s t, t s t, \underline{w}, s t s$, sts).

Finally suppose that $\left(d_{0}, \ldots, d_{4}\right)$ is as in (4). In this case the image under $\delta_{c}$ of conv $\left\{d_{0}, d_{4}\right\}$ is not uniquely determined by $\left(d_{0}, \ldots, d_{4}\right)$, the two cases in $\left(4^{\prime}\right)$ being possible. In the first case a careful look at the whole set of chambers shows that $\operatorname{conv}\left\{d_{0}, d_{4}\right\} \in\left(\mathscr{A}^{\Phi_{s t s} \cap \Phi_{t s t}}\right)^{\prime}$. Note also that in no other case, except the one already examined, does $\delta_{c}$ map conv $\left\{d_{0}, d_{4}\right\}$ onto $\Phi_{s t s} \cap \Phi_{t s t}$. So $\left(\mathscr{A}^{\Phi_{s s t} \cap \Phi_{t s t}}\right)^{\prime}=\mathscr{A}^{\Phi_{s t s} \cap \Phi_{s t}}$. In the second case we have again that $\delta_{c}$ maps $\operatorname{conv}\left\{d_{0}, d_{4}\right\}$ onto $\Phi_{t} \cap \Phi_{s t s}$, but, as the cardinality of the fiber $\delta_{c}^{-1}(\underline{w}) \cap \operatorname{conv}\left\{d_{0}, d_{4}\right\}$ is different from the other case, the set of apartments that we obtain is disjoint from $\left(\mathscr{A}^{\Phi_{s i s} \cap \Phi_{1}}\right)^{\prime}$. Denote by $\left(\mathscr{A}^{\Phi_{s t s} \cap \Phi_{1}}\right)^{\prime \prime}$ this new set of apartments. As each apartment in $\left(\mathscr{A}^{\Phi_{s s} \cap \Phi_{t}}\right)^{\prime \prime}$ contains two galleries as in (4) while each apartment in $\mathscr{A}^{\Phi_{s t s} \cap \Phi_{s t}}$ contains only one such gallery, then (4.6) follows from the equality

$$
2\left|\left(\mathscr{A}^{\Phi_{s s s} \cap \Phi_{t}}\right)^{\prime \prime}\right|=\left|\Gamma_{t, s . t . s}(t s t, t s t, \underline{w}, \underline{w}, \underline{w})\right|-\left|\Gamma_{t . s . t . s}(t s t, t s t, \underline{w}, s t s, s t s)\right| .
$$

To conclude our proof note that, by symmetry, we obtain formulas like (4.4), (4.5) and (4.6) by exchanging the roles of $s$ and $t$.

REMARK 4.7. If $A$ is a fixed apartment in any building $\mathbf{X}$ with $c \in \mathscr{C}(A)$ as fundamental chamber and if $\left\{\Phi_{i}\right\}_{i \in I}$ is the set of negative half-apartments in $A$ corresponding to the set of simple reflections, then writing $d \in \bigcap_{i \in I} \Phi_{i}$ is the same as stating that $d$ is opposite to $c$ [9, Theorem 2.16].

Let now $\mathbf{X}$ be a spherical building which is the direct product $\left[9\right.$, p. 33] $\mathbf{X}_{1} \times \cdots \times \mathbf{X}_{n}$ and let $A=A_{1} \times \cdots \times A_{n}$ and $c=\left(c_{1}, c_{2}, \ldots, c_{n}\right)$ as above. Moreover, for $1 \leq k \leq n$ let $I_{k}$ be the set of types of $\mathbf{X}_{k}$ and $\left\{\Phi_{i_{k}}\right\}_{i_{k} \in I_{k}}$ the corresponding set of negative halfapartments in $A_{k}$. As for a chamber $d=\left(d_{1}, \ldots, d_{n}\right)$ one has that $\mathbf{d}(c, d)=\operatorname{diam} \mathbf{X}$ if and only if $\mathbf{d}\left(c_{k}, d_{k}\right)=\operatorname{diam} \mathbf{X}_{k}$ for $1 \leq k \leq n$, then the following equality holds

$$
\left|\mathscr{A}^{n_{i \epsilon} \Phi_{i}}\right|=\left|\mathscr{A}_{1}^{\cap_{i \in l_{1}} \Phi_{i_{1}}}\right| \cdots\left|\mathscr{A}_{n}^{n_{i n} \in \epsilon_{n} \Phi_{i_{n}}}\right| .
$$

REMARK 4.8. By Proposition 4.1, writing $\left\{A \cap l k(\sigma): A \in \mathscr{A}_{\sigma}^{\Phi}\right\}$ is the same as writing $\left\{A \cap l k(\sigma): A \in \mathscr{A}_{\sigma}\right\}^{\Phi \cap W_{\sigma}}$. 
In the sequel we will simply write $m$ for $m_{Q}$.

COROLLARY 4.9. Let $\mathbf{X}$ an affine building with diagram of type $\widetilde{A_{2}}$ and parameter $q$. For each $i, j=0,1,2$ such that $i<j$ the following equalities hold:

$$
\begin{aligned}
m\left(\mathscr{A}^{\Phi_{i}}\right) & =\frac{(q-1)}{2}, \\
m\left(\mathscr{A}^{\Phi_{i j i}}\right) & =\frac{(q-1)}{2}, \\
m\left(\mathscr{A}^{\Phi_{i} \cap \Phi_{i j i}}\right) & =m\left(\mathscr{A}^{\Phi_{j} \cap \Phi_{i j i}}\right)=\frac{(q-1)^{2}}{2} .
\end{aligned}
$$

PROOF. First note that if $\sigma=\partial \Phi \cap c$ then $\mathscr{A}^{\Phi}=\mathscr{A}_{\sigma^{\prime}}^{\Phi}$ for each $\sigma^{\prime} \subseteq \sigma$. Then, as $\sigma_{i}=\partial \Phi_{i} \cap c$ and as $l k\left(\sigma_{i}\right)$ is a building of rank one (and diameter one), (4.7) follows from (2.2) and from Lemma 4.2. Analogously, for each fixed $i, j$, if $x$ is the vertex of $c$ of type $I \backslash\{i, j\}$ then $l k(x)$ is of type $A_{2}$ and $\partial \Phi_{i}, \partial \Phi_{j}, \partial \Phi_{i j i}$ are the three walls containing $x$. Therefore (4.8), (4.9) follow easily respectively from (4.2), (4.3) and from (2.2).

COROLLARY 4.10. Let $\mathbf{X}$ be an affine building with diagram of type $\widetilde{B}_{2}$ and suppose that the vertex of clabelled by $x_{0}$ is such that $l k\left(x_{0}\right)$ has diagram of type $A_{1} \times A_{1}$. If $i \in I \backslash\{0\}$, then the following formulas hold:

$$
\begin{aligned}
m\left(\mathscr{A}^{\Phi_{i}}\right) & =\frac{\left(q_{i}-1\right)}{2}, \\
m\left(\mathscr{A}^{\Phi_{0 i 0}}\right) & =\frac{\left(q_{i}-1\right)}{2}, \\
m\left(\left(\mathscr{A}^{\Phi_{0 i 0} \cap \Phi_{i}}\right)^{\prime}\right) & =\frac{\left(q_{i}-1\right)^{2}}{4}, \\
m\left(\left(\mathscr{A}^{\Phi_{0 i 0} \cap \Phi_{i}}\right)^{\prime \prime}\right) & =\frac{\left(q_{0}-1\right)\left(q_{i}-1\right)\left(q_{i}-2\right)}{2} .
\end{aligned}
$$

Moreover

$$
\begin{aligned}
m\left(\mathscr{A}^{\Phi_{1} \cap \Phi_{2}}\right) & =\frac{\left(q_{1}-1\right)\left(q_{2}-1\right)}{4}, \\
m\left(\mathscr{A}^{\Phi_{010} \cap \Phi_{020}}\right) & =\frac{q_{0}\left(q_{1}-1\right)\left(q_{2}-1\right)}{4} .
\end{aligned}
$$

PROOF. To prove (4.10) we argue as in the proof of (4.7). Fix $i \in I \backslash\{0\}$ and let $j \in I \backslash\{0, i\}$. As $l k\left(x_{j}\right)$ is of type $B_{2}$ then (4.11), (4.12), (4.13) follow respectively 
from (4.4), (4.5), (4.6), with $s_{0}=s$ and $s_{i}=t$. To prove (4.14) note that $\left\{s_{i}\right\}_{i=1.2}$ is the set of simple reflections in $W_{x_{0}}$. Then $\mathscr{A}^{\Phi_{1} \cap \Phi_{2}}=\mathscr{A}_{x_{0}}^{\Phi_{1} \cap \Phi_{2}}$ and

$$
m\left(\mathscr{A}^{\Phi_{1} \cap \Phi_{2}}\right)=\left|\left\{A \cap l k\left(x_{0}\right): A \in \mathscr{A}_{x_{0}}\right\}^{\left(\Phi_{1} \cap \Phi_{2}\right) \cap w_{x_{0}}}\right|\left(q_{1} q_{2}\right)^{-1}
$$

As $l k\left(x_{0}\right)$ is the direct product of two spherical buildings of rank one then (4.14) follows from Remark 4.7 and from Lemma 4.2 (with $s=s_{i}$ ).

To prove (4.15) note that as $s_{0}$ interchanges $\Phi_{0}$ and $\Phi_{0}^{+}$we have that $\Phi_{0 i 0}=s_{0} \Phi_{i}$, $i=1,2$. In other words, $\Phi_{010}$ and $\Phi_{020}$ are the negative half-apartments which correspond to the simple reflections in $s_{0} W_{x_{0}} s_{0}=W_{s_{0} x_{0}}$ and hence $\Phi_{010} \cap \Phi_{020}=\{d \in$ $\left.\mathscr{C}\left(A_{0}\right): d\left(\operatorname{proj}_{s_{0} x_{0}} d, s_{0} c\right)=\operatorname{diam} l k\left(s_{0} x_{0}\right)\right\}$. Denote by $c_{k}, 1 \leq k \leq q_{0}$, the generic chamber in $\mathbf{X} 0$-adjacent to $c$, and let $x_{0}^{k}$ be its vertex of type 0 . Then $\rho\left(c_{k}\right)=s_{0} c$ and $\rho\left(x_{0}^{k}\right)=s_{0} x_{0}$. Now we show that for each $A \in \mathscr{A}^{\Phi_{010} \cap \Phi_{020}}$ there exists a unique $k \in\left\{1, \ldots, q_{0}\right\}$ such that for each $d \in \mathscr{C}(A) \operatorname{proj}_{\sigma_{0}} d=c_{k}$ and $x_{0}^{k} \in A$. First note that $\Phi_{010} \cap \Phi_{020} \subset \Phi_{0}$. In fact if $d \in \Phi_{010} \cap \Phi_{020}$ then $d \notin \Phi_{0}^{+}$, otherwise $d$ would be opposite to $s_{0} c$, contradicting the fact that $\mathbf{X}$ is affine [11, Theorem 2.36]. Hence, if $A \in \mathscr{A}^{\Phi_{010} \cap \Phi_{020}}$, for each $d \in \mathscr{C}(A)$ then $\operatorname{proj}_{\sigma_{0}} d=c_{k}$ for some $k$, (otherwise $\operatorname{proj}_{\sigma_{0}} d=c$ and $\rho(d) \in \operatorname{proj}_{\sigma_{0}}^{-1} c \cap A_{0}=\Phi_{0}^{+}$). Moreover if $d$ and $d^{\prime}$ are two distinct chambers in $A$ such that $\operatorname{proj}_{\sigma_{0}} d \neq \operatorname{proj}_{\sigma_{0}} d^{\prime}$, then for the convexity of apartments we have that $\sigma_{0} \in A$ and then $\rho(A)=\Phi_{0}$ which strictly contains $\Phi_{010} \cap \Phi_{020}$.

As the retraction $\rho$ preserves $i$-adjacency and the distance from $c$ it follows that

$$
\left\{A \in \mathscr{A}_{x_{0}^{k}}: \forall d \in \mathscr{C}(A) \quad d\left(\operatorname{proj}_{x_{0}^{k}} d, c_{k}\right)=\operatorname{diam} l k\left(x_{0}^{k}\right)\right\}=\mathscr{A}_{x_{0}^{k}}^{\Phi_{010} \cap \Phi_{020}},
$$

and

$$
\mathscr{A}^{\Phi_{010} \cap \Phi_{020}}=\bigcup_{1 \leq k \leq q_{0}} \mathscr{A}_{x_{0}^{k}}^{\Phi_{010} \cap \Phi_{020}},
$$

where the union is disjoint.

Moreover for each $1 \leq k \leq q_{0}$

$$
m\left(\mathscr{A}_{x_{0}^{k}}^{\Phi_{010} \cap \Phi_{020}}\right)=m\left(\mathscr{A}_{s_{0} x_{0}}^{\Phi_{010} \cap \Phi_{020}}\right)=m\left(\mathscr{A}_{x_{0}}^{\Phi_{1} \cap \Phi_{2}}\right),
$$

and we get (4.15) by taking the sum over $k$.

Let now $\mathbf{X}$ be a building with diagram of type $\widetilde{A_{2}}$. We introduce the bounded operator $M: L^{\infty}(\mathscr{A}) \rightarrow L^{\infty}(\mathscr{C})$ which we obtain by radializing with respect to $m$ the inversion formula which follows from the application of Lemma 3.1 to the right-hand 
side of (3.2): for each $\varphi \in L^{\infty}(\mathscr{A})$ we set

$$
\begin{aligned}
M \varphi(c)= & \left\{\int_{\mathscr{A}^{\wedge} 0}-\sum_{0 \leq i \leq 2} \frac{1}{(q-1)} \int_{\mathscr{A}^{\Phi_{i}}}-\sum_{0 \leq i<j \leq 2}\left[\frac{1}{3(q-1)} \int_{\mathscr{A}^{\Phi_{i j i}}}\right.\right. \\
& \left.\left.-\frac{1}{3(q-1)^{2}}\left(\int_{\mathscr{A}^{\Phi_{i} \cap \Phi_{i j i}}}+\int_{\mathscr{A}^{\Phi_{j} \cap \boldsymbol{\Phi}_{i j i}}}\right)\right]\right\} \varphi(A) d m(A) .
\end{aligned}
$$

Formulas (4.7), (4.8), (4.9) show indeed that $M \varphi \in L^{\infty}(\mathscr{C})$ and that $\|M\| \leq 4$.

LEMMA 4.11. Let $\mathbf{X}$ be a building with diagram of type $\widetilde{A_{2}}$ and let $d \in \mathscr{C}$. If $\rho(d) \in \Phi$ and $i, j \in I, i<j$ then

$$
\begin{aligned}
& q^{\mathrm{d}(c . d)} m\left(\mathscr{A}^{\Phi} \cap \mathscr{A}_{d}\right)= \\
&= \begin{cases}1 & \text { if } \Phi=A_{0} ; \\
(q-1) & \text { if } \Phi=\Phi_{i} ; \\
(q-1) & \text { if } \Phi=\Phi_{i j} ; \\
(q-1)^{2} & \text { if } \Phi=\Phi_{i} \cap \Phi_{i j i} \text { or } \Phi=\Phi_{j} \cap \Phi_{i j i}, \rho(d) \notin \Phi_{i} \cap \Phi_{j} ; \\
2(q-1)^{2} & \text { if } \Phi=\Phi_{i} \cap \Phi_{i j i} \text { or } \Phi=\Phi_{j} \cap \Phi_{i j i}, \rho(d) \in \Phi_{i} \cap \Phi_{j} .\end{cases}
\end{aligned}
$$

PROOF. If $\Phi=A_{0}$ then, as $\mathscr{A}^{A_{0}}=\mathscr{A}_{c}$, the assertion follows immediately from the definition of $m$. In the other cases let $\sigma=\partial \Phi \cap c$. As $\rho(d) \in \Phi$ if and only if $\rho\left(\operatorname{proj}_{\sigma}\right) \in \Phi$, we can argue by induction on the length of a minimal gallery from $\sigma$ to $d$. This suffices to prove the assertion for $d \in \mathscr{C}(l k(\sigma))$.

Denote by $n(\sigma, \Phi, d)$ the number of apartments in $\left\{A \cap l k(\sigma): A \in \mathscr{A}^{\Phi}\right\}$ containing $d$, and let $w \in W_{\sigma}$ be such that $\delta_{c}(d)=w$. Then as for each $E \in$ $\left\{A \cap l k(\sigma): A \in \mathscr{A}^{\Phi}\right\}$

$$
n(\sigma, \Phi, d)=\frac{\left|\left\{A \cap l k(\sigma): A \in \mathscr{A}^{\Phi}\right\}\right|\left|\delta_{c}^{-1}(w) \cap \mathscr{C}(E)\right|}{\left|\delta_{c}^{-1}(w)\right|},
$$

we have

$$
m\left(\mathscr{A}^{\Phi} \cap \mathscr{A}_{d}\right)=\frac{n(\sigma, \Phi, d)}{N\left(1, W_{\sigma}\right)}=\frac{m\left(\mathscr{A}^{\Phi}\right)\left|\delta_{c}^{-1}(w) \cap \mathscr{C}(E)\right|}{\left|\delta_{c}^{-1}(w)\right|} .
$$

As $\mathbf{X}$ is homogeneous we have that $\left|\delta_{c}^{-1}(w)\right|=q^{\mathbf{d}(c, d)}$. Then, case by case, by Corollary 4.9, to complete the proof we have to look only for the value of $\mid \delta_{c}^{-1}(w) \cap$ $\mathscr{C}(E) \mid$. If $\Phi=\Phi_{i}$, then $\sigma=\sigma_{i}$. The assertion follows as $\delta_{c}$ maps each of the two chambers, of each apartment in $\left\{A \cap l k(\sigma): A \in \mathscr{A}^{\Phi}\right\}$, onto $\left\{s_{i}\right\}=\Phi_{i} \cap W_{\sigma_{i}}$. If $\Phi=\Phi_{i j i}, \Phi_{i j i} \cap \Phi_{i}$ or $\Phi_{i j i} \cap \Phi_{j}$, then $\sigma$ is equal to the vertex $x$ of $c$ of type $I \backslash\{i, j\}$. In each of these cases for each $w \in W_{x} \cap \Phi$ we can recover the value of 
$\left|\delta_{c}^{-1}(w) \cap \mathscr{C}(E)\right|$ from the discussion in the proof of Lemma 4.5 where we have to put $s=s_{i}$ and $t=s_{j}$. Note only that writing $\rho(d) \in \Phi_{i} \cap \Phi_{j}$ is another way of stating that $\delta_{c}(d)=s_{i} s_{j} s_{i}=s_{j} s_{i} s_{j}$.

Proposition 4.12. Let $\mathbf{X}$ be a building with diagram of type $\widetilde{A_{2}}$ and let $g \in$ $L^{1}(\mathscr{C}(A))$ for each $A \in \mathscr{A}$. Then

$$
M R g=g
$$

PROOF. For fixed $c$ in $\mathscr{C}$ we will prove (4.17) at $c$. If $d$ is any chamber in $\mathbf{X}$ denote by $\mathbf{1}_{d}$ the Dirac function in $d$. As $d$ ranges in $\mathscr{C}$, the set $\left\{\mathbf{1}_{d}\right\}$ is a basis for the space of functions that are summable on each apartment of $\mathbf{X}$. Then for the linearity of $M R$ it will be sufficient to prove that for each $d \in \mathscr{C},(4.17)$ holds when $g=\mathbf{1}_{d}$.

If $d=c$ then we use (4.16) with $\Phi=A_{0}$ to prove that each side of (4.17) is equal to 1. Let now $d$ be any chamber distinct from $c$, then, up to considering its image under the retraction $\rho_{c, A_{0}}$, we can suppose that $d \in \mathscr{C}\left(A_{0}\right)$. Therefore $d \in \Phi_{k}$ for some $k \in I$. Note that from [11, Proposition 2.32] this holds if and only if $\operatorname{proj}_{\sigma_{k}} d=s_{k} c$. Then, denoting by $x_{k^{-}}$and $x_{k^{+}}$the vertices of $c$ respectively of type $k-1 \bmod 3$ and $k+1 \bmod 3$, as $\sigma_{k}=\left(x_{k^{-}}, x_{k^{+}}\right)$and as proj $j_{k_{k^{ \pm}}}$is contained in the convex hull of $\sigma_{k}$ and $d$, we have that for $d \in \Phi_{k}$ only the following cases are possible:

(1) $\operatorname{proj}_{x_{k^{-}}} d=\operatorname{proj}_{\sigma_{k}} d=\operatorname{proj}_{x_{k^{+}}} d$;

(2) $\operatorname{proj}_{x_{k^{-}}} d=\operatorname{proj}_{\sigma_{k}} d \neq \operatorname{proj}_{x_{k^{+}}} d$;

(3) $\operatorname{proj}_{x_{k^{-}}} d \neq \operatorname{proj}_{\sigma_{k}} d=\operatorname{proj}_{x_{k^{+}}} d$;

(4) $\operatorname{proj}_{x_{k^{-}}} d \neq \operatorname{proj}_{\sigma_{k}} d$ and $\operatorname{proj}_{x_{k^{+}}} d \neq \operatorname{proj}_{\sigma_{k}} d$.

As $s_{k}$ interchanges $\Phi_{k}$ and $\Phi_{k}^{+}$, then applying again [11, Proposition 2.32] we obtain that

$$
\operatorname{proj}_{x_{k^{-}}}^{-1}\left(s_{k} C\right)=s_{k} \Phi_{k^{+}}^{+} \cap s_{k} \Phi_{k}^{+}=\Phi_{k k^{+} k}^{+} \cap \Phi_{k}
$$

and

$$
\operatorname{proj}_{x_{k^{+}}}^{-1}\left(s_{k} C\right)=s_{k} \Phi_{k^{-}}^{+} \cap s_{k} \Phi_{k}^{+}=\Phi_{k k^{-} k}^{+} \cap \Phi_{k}
$$

If $d$ satisfies the equalities in (1) then $d \in \operatorname{proj}_{x_{k^{-}}}^{-1}\left(s_{k} C\right) \cap \operatorname{proj}_{x_{k^{+}}}^{-1}\left(s_{k} C\right)$ and then $d=s_{k} c=\cap_{i \in I} s_{k} \Phi_{i}^{+}$. Hence we can use (4.16) with $\Phi=A_{0}$ and $\Phi=\Phi_{k}$ to prove that each side of (4.17) vanishes. If $d$ satisfies (2), then being $d \in s_{k} \Phi_{k^{+}}^{+} \cap s_{k} \Phi_{k}$ and $d \notin s_{k} \Phi_{k^{-}}^{+} \cap s_{k} \Phi_{k}$ we have that $d \in s_{k} \Phi_{k^{-}} \cap \Phi_{k}$, and then we use (4.16) with $\Phi=A_{0}, \Phi_{k}$ and $\Phi_{k k^{-k}}$ (considering the two cases $d \in \Phi_{k} \cap \Phi_{k^{-}}$and $d \notin \Phi_{k} \cap \Phi_{k^{-}}$) to conclude that also in this case each side of (3.19) is equal to 0 . Case (3) is symmetric to (2), so we obtain the same result by arguing as above with $k^{-}$replaced by $k^{+}$. Finally, the inequalities in (4) imply that $d \in \Phi_{k k^{-k}} \cap \Phi_{k k^{+} k}$. Observe that 
the wall $\partial \Phi_{k k^{-k}}$ (respectively $\partial \Phi_{k k^{+} k}$ ) is parallel to $\partial \Phi_{k^{+}}$(respectively $\partial \Phi_{k^{-}}$). Hence $\Phi_{k k^{-k}} \subset \Phi_{k^{+}}^{+}$, (respectively $\Phi_{k k^{+} k} \subset \Phi_{k^{-}}^{+}$). Then if $d \in \Phi_{k k^{-k}} \cap \Phi_{k k^{+} k}$ it cannot happen that $d \in \Phi_{k} \cap \Phi_{k^{-}}$or $d \in \Phi_{k} \cap \Phi_{k^{+}}$and we use (4.16) with $\Phi=A_{0}, \Phi_{k}, \Phi_{k k^{-k}}$ and $\Phi_{k k^{+} k}\left(d \notin \Phi_{k} \cap \Phi_{k^{-}}\right.$and $\left.d \notin \Phi_{k} \cap \Phi_{k^{+}}\right)$to prove that each side of (4.17) equals 0 and hence to conclude the proof.

Let now $\mathbf{X}$ be a building of type $\widetilde{B_{2}}$. In analogy with the previous case we introduce the bounded operator $M: L^{\infty}(\mathscr{A}) \rightarrow L^{\infty}(\mathscr{C})$ defined by

$$
\begin{aligned}
& M \varphi(c)=\left\{\int_{\mathscr{A}^{\Lambda_{0}}}-\sum_{i=1,2}\left[\frac{1}{\left(q_{i}-1\right)}\left(\int_{\mathscr{A}^{\Phi_{i}}}+\int_{\mathscr{S}^{\Phi_{0 i \mathrm{i}}}}\right)-\frac{1}{\left(q_{i}-1\right)^{2}} \int_{\left(\mathscr{A}^{\Phi_{i}} \cap \oplus_{\phi_{0 i}}\right)^{\prime}}\right]\right. \\
& \left.+\frac{1}{\left(q_{1}-1\right)\left(q_{2}-1\right)} \int_{\mathscr{\infty}^{\phi_{1} \cap \phi_{2}}}+\frac{1}{q_{0}\left(q_{1}-1\right)\left(q_{2}-1\right)} \int_{\mathscr{S}^{\Phi_{010} \cap \oplus_{020}}}\right\} \varphi(A) d m(A) \text {. }
\end{aligned}
$$

Denote by $T_{i}, i=0,1,2$ the operator defined on the space of complex-valued functions on $\mathscr{C}$, by

$$
T_{i} f(c)=\frac{1}{q_{i}} \sum_{\substack{c^{\prime} \sim c \\ c^{\prime} \neq c}} f\left(c^{\prime}\right)
$$

PROPOSITION 4.13. For each $f \in L^{1}(\mathscr{C}(A))$ we get

$$
M R f=\left(1+T_{0}\right) f .
$$

Before proving Proposition 4.13 we need the following

LEMMA 4.14. Let $d \in \mathscr{C}$ and $\rho(d) \in \Phi \subset A_{0}$ then

(4.19) $\left|\delta_{c}^{-1}\left(\delta_{c}(d)\right)\right| m\left(\mathscr{A}^{\Phi} \cap \mathscr{A}_{d}\right)= \begin{cases}1 & \text { if } \Phi=A_{0} ; \\ \left(q_{i}-1\right) & \text { if } \Phi=\Phi_{i}, i=1,2 ; \\ \left(q_{i}-1\right) & \text { if } \Phi=\Phi_{0 i 0}, i=1,2 ; \\ \left(q_{1}-1\right)\left(q_{2}-1\right) & \text { if } \Phi=\Phi_{1} \cap \Phi_{2} ; \\ q_{0}\left(q_{1}-1\right)\left(q_{2}-1\right) & \text { if } \Phi=\Phi_{010} \cap \Phi_{020} .\end{cases}$

Moreover if $\Phi=\Phi_{i} \cap \Phi_{0 i 0}$ then

$$
\left|\delta_{c}^{-1}\left(\delta_{c}(d)\right)\right| m\left(\left(\mathscr{A}^{\Phi}\right)^{\prime} \cap \mathscr{A}_{D}\right)=\left(q_{i}-1\right)^{2} .
$$

PROOF. We use essentially the same argument as in the proof of Lemma 4.11. So in the same way we prove the assertion when $\Phi=A_{0}$ and $\Phi=\Phi_{i}$. Similarly we can prove (4.19) when $\Phi=\Phi_{0 i 0}$ and (4.20) (apply Corollary 4.10 and see respectively 
the proof of (4.4) and (4.5) to find out the cardinality of the fiber $\delta_{c}^{-1}(w) \cap A$ for each $w \in \Phi$ and $A \in \mathscr{A}^{\Phi}$ ). When $\Phi=\Phi_{1} \cap \Phi_{2}$ the assertion follows again from Corollary 4.9 and the fact that all four chambers in $W_{x_{0}}=\left\{1, s_{1}, s_{2}, s_{1} s_{2}=s_{2} s_{1}\right\}$ retract onto $\left\{s_{1} s_{2}\right\}$. When $\Phi=\Phi_{010} \cap \Phi_{020}$ then we know from Corollary 4.10 that for any fixed chamber $d$ such that $\rho(d) \in \Phi$, there exist a unique chamber $c_{k}, k \in\left\{1, \ldots, q_{0}\right\}$, 0 -adjacent to $c$ such that $\mathbf{d}\left(c_{k}, d\right)=\mathbf{d}(c, d)-1$, (indeed $\left.\operatorname{proj}_{\sigma_{0}} d\right)$, and such that if $x_{0}^{k}$ is its vertex of type 0 , then

$$
\mathscr{A}^{\Phi} \cap \mathscr{A}_{d}=\mathscr{A}_{x_{0}^{i}}^{\Phi} \cap \mathscr{A}_{d}
$$

Then by symmetry with the case $\Phi=\Phi_{1} \cap \Phi_{2}$ the assertion follows as $\left|\delta_{c}^{-1}\left(\delta_{c}(d)\right)\right|=$ $q_{0}\left|\delta_{c_{k}}^{-1}\left(\delta_{c_{k}}(d)\right)\right|$.

PROOF OF PROPOSITION 4.13. We proceed and use the same notation as in the proof of Proposition 4.12. Note that, as $\left\{c, s_{0} c\right\}=\left(\cap_{i=1,2} \Phi_{i}^{+}\right) \cap\left(\cap_{i=1.2} \Phi_{0 i 0}^{+}\right)$then $\mathscr{C}\left(A_{0}\right) \backslash$ $\left\{c, s_{0} c\right\}=\left(\cup_{i=1,2} \Phi_{i}\right) \cup\left(\cup_{i=1,2} \Phi_{0 i 0}\right)$. Note also that, as $\partial \Phi_{1}$ is parallel to $\partial \Phi_{020}$ and $\partial \Phi_{2}$ is parallel to $\partial \Phi_{010}$, then $\Phi_{1} \cap \Phi_{020}=\Phi_{2} \cap \Phi_{010}=\emptyset$. Then if $f=\mathbf{1}_{c}$ (respectively $f=1_{s_{0} c}$ ) we can use (4.19) only in the case $\Phi=A_{0}$ to prove that each side of (4.18) is equal to 1 (respectively to $\left(q_{0}\right)^{-1}$ ). Let $f=\mathbf{1}_{d}, d \in \mathscr{C}\left(A_{0}\right) \backslash\left\{c, s_{0} c\right\}$ and suppose for example that $d \in \Phi_{1}$. Then, as one can easily verify, only the following three cases can occur:

(1) $d \in \Phi_{2}$

(2) $d \in \Phi_{010}$;

(3) $d \in \Phi_{2}^{+} \cap \Phi_{010}^{+}$.

In the first case we use (4.19) with $\Phi=\Phi_{1}$ and $\Phi=\Phi_{1} \cap \Phi_{2}$ to prove that each side of (4.18) vanishes. In the second case we use (4.19) with $\Phi=\Phi_{1}, \Phi=\Phi_{010}$ and (4.20) with $i=1$ and we obtain again that each side of (4.18) is equal to 0 . Finally in the third case we can use (4.19) only in the case $\Phi=\Phi_{1}$ to conclude that also in this case each side of (4.18) is equal to 0 .

Similarly one can argue in the other possible cases $\left(d \in \Phi_{2}, \ldots\right)$, and applying Lemma 4.14 one proves that (4.18) holds for $f=1_{d}$ for each $d \in \mathscr{C}\left(A_{0}\right)$ (then in X). By linearity and continuity, (4.18) follows for an arbitrary $f$.

LemMa 4.15. For $i=0,1,2,\left(1+T_{i}\right)^{-1}$ is given by

$$
\frac{1}{2\left(q_{i}-1\right)}\left[\left(2 q_{i}-1\right)-q_{i} T_{i}\right]
$$

PROOF. First prove that $T_{i}^{2}=\left(1 / q_{i}\right)+\left(1-1 / q_{i}\right) T_{i}$. Then the assertion follows by direct computation.

We have just proved the following proposition. 
PROPOSITION 4.16. Let $\mathbf{X}$ be any building of type $\widetilde{B}_{2}$. Then the Radon transform $R$ is inverted by

$$
\frac{1}{2\left(q_{0}-1\right)}\left[\left(2 q_{0}-1\right)-q_{0} T_{0}\right] M R f=f
$$

REMARK 4.17. Applying the same techniques, an inversion formula of the same kind of $(4.21)$ can be obtained for a building with diagram of type $\widetilde{G_{2}}$. Moreover the existence of simpler and more general inversion formulas can be proved [3] for 'large enough' values of parameters.

\section{References}

[1] G. Ahumada Bustamante, Analyse harmonique sur l'espace des chemins d'un arbre, Tesi di dottorato di Stato, Università di Parigi Sud, Orsay, 1988.

[2] L. Atanasi, Trasformate di Radon su edifici affini, Tesi di dottorato, Università di Roma 'La Sapienza', 1996.

[3] ——, 'Radon transforms on $\widetilde{A_{n}}$-buildings', preprint, (Università di Roma 'Tor Vergata', 1997).

[4] C. A. Berenstein, E. Casadio Tarabusi, J. M. Cohen and M. Picardello, 'Integral geometry on trees', Amer. J. Math. 113 (1991), 441-470.

[5] N. Bourbaki, Integration (Hermann, Paris, 1959).

[6] K. S. Brown, Buildings (Springer, New York, 1989).

[7] R. W. Carter, Simple groups of Lie type (Wiley-Interscience, New York, 1962).

[8] J. E. Humphreys, Reflection groups and Coxeter groups (Cambridge, 1990).

[9] M. Ronan, Lectures on buildings (Academic Press, Boston, 1990).

[10] T. Steger, personal communication.

[11] J. Tits, Buildings of spherical type and finite BN-pairs, Lecture Notes in Mathematics 386 (Springer, 1974).

[12] — 'A local approach to buildings', in: The geometric vein. The Coxeter Festschrift (Springer, New York, 1981) pp. 519-547.

Department of Mathematics

University of Rome 'Tor Vergata'

00135 Rome

Italy

e-mail: atanasi@axp.mat.uniroma2.it 